\title{
Role of continuum in nuclear direct reactions with one-neutron halo nuclei: A one-dimensional model
}

\author{
L. Moschini $\odot,{ }^{1,}{ }^{*}$ A. M. Moro $\odot,{ }^{2}$ and A. Vitturi $\odot^{3,4}$ \\ ${ }^{1}$ Department of Physics, Faculty of Engineering and Physical Sciences, University of Surrey, Guildford, \\ Surrey GU2 7XH, England, United Kingdom \\ ${ }^{2}$ Departamento de Física Atómica, Molecular y Nuclear, Universidad de Sevilla, Apartado 1065, E-41080 Sevilla, Spain \\ ${ }^{3}$ Dipartimento di Fisica e Astronomia “G. Galilei”, Università degli Studi di Padova, Via Marzolo 8, 35131 Padova, Italy \\ ${ }^{4}$ Istituto Nazionale di Fisica Nucleare, Sezione di Padova, Via Marzolo 8, 35131 Padova, Italy
}

(Received 11 September 2020; accepted 21 December 2020; published 8 January 2021)

\begin{abstract}
Background: The problem of the scattering of a one-neutron halo nucleus by another nucleus might involve an extremely complicated solution, particularly when breakup and rearrangement channels are to be considered.

Purpose: We construct a simple model to study the evolution of a single-particle wave function during the collision of a one-dimensional potential well by another well.

Method: Our one-dimensional model provides the essential three-body nature of this problem, and allows for a much simpler application and assessment of different methods of solution. To simplify further the problem, we assume that the potential well representing the projectile moves according to a predetermined classical trajectory, although the internal motion of the "valence" particle is treated fully quantum mechanically. This corresponds to a semiclassical approach of the scattering problem, applicable in the case of heavy projectile and target. Different approaches are investigated to understand the dynamics involving one-body halo-like systems: the "exact" time-dependent solution of the Schrödinger equation is compared to a numerical continuum-discretized coupled-channels (CC) calculation presenting various model cases including different reaction channels.

Results: This framework allows us to discuss the reaction mechanism and the role of the continuum, the inclusion of which in the $\mathrm{CC}$ calculation results to be crucial to reproduce the exact solution, even when the initial and final states are well bound.

Conclusions: The dynamical situations under study can be linked to analogous problems solved in a threedimensional (3D) CC framework, so the present model provides a simple tool to understand the main challenges experienced in the usual 3D models with the treatment of the continuum.
\end{abstract}

DOI: 10.1103/PhysRevC.103.014604

\section{INTRODUCTION}

One of the most relevant research lines in contemporary nuclear physics is the investigation, both experimentally and theoretically, of nuclei under extreme conditions and, in particular, nuclei far from the stability valley. Along this line, truly enticing and sometimes striking novel nuclear structure phenomena are being observed. In particular, these so-called exotic nuclei present low proton or neutron separation energy, short lifetime, and a radius which noticeably deviates from the $A^{1 / 3}$ dependence of stable nuclei, a fact that is related to a skin or a halo structure $[1,2]$. The excess of neutrons or protons also leads to a different arrangement which is generally described as deformation: this is indicated by new single-particle character of states and new magic numbers related to new shell closure [3,4]. Because of the large spatial separation between the center of mass and the center of charge, in presence of an external electric field low-energy electric dipole excitations can result, as well as very clear

*1.moschini@surrey.ac.uk cluster effects [5]. Examples of exotic systems are nuclei with large neutron excess, with the barely bound outermost ones creating an extended density distribution, referred to as a halo. There can be different kinds of halo systems: a nucleus with one valence neutron is for example ${ }^{11} \mathrm{Be}$, whereas ${ }^{8} \mathrm{~B}$ is an isotope presenting a one-proton halo.

Nowadays, measuring the properties of such nuclei is the goal of the main experimental nuclear facilities around the world $[6,7]$. Due to their short lifetimes targets of such nuclei are not feasible, so they cannot be studied by usual spectroscopic techniques. Thus, the challenge to measure nuclei on the drip lines has triggered the development of radioactive nuclear beams [8]. Most of our present knowledge of stable and exotic nuclei stems from the analysis of nuclear direct reactions. Direct reactions are characterized by different channels-elastic and inelastic scattering, transfer of nucleons between the colliding nuclei, and breakup (i.e., excitation to positive energy states) - and they will feed a particular channel in a way that depends sensitively on its character [9]. In particular, inelastic scattering excites collective states strongly; one-neutron transfer probes the single-particle character of states; two-nucleon transfer goes preferentially to 
states that exhibit strong pairing correlations; pairing could also be tested via multinucleon transfer, as well as clustering; the role of the continuum is investigated through breakup reactions.

From a theoretical point of view, the conventional theories valid for the description of stable nuclei have difficulties to describe exotic systems, thus indicating the necessity to improve them and include the description of the new phenomena taking place at the limits of stability, taking into account the new features. The theoretical description of halo nuclei is strongly characterized by its weakly bound nature: the valence nucleons are so weakly bound that the addition of any correlation to the simple mean field model inevitably involves the inclusion of the continuum in the system description. For this reason the description of their structure or dynamics is more involved, even considering inert cores. Direct reactions are usually studied applying coupled-channels (CC) calculations; in the case of processes involving exotic systems the continuum discretized coupled-channels (CDCC) description is applied [10-13]. However, it is often difficult to model the continuum, e.g., when transfer or breakup channels are dominant [14-16]. Time-dependent approaches are often used to disentangle the reaction mechanism (see, e.g., Refs. [17-20]) and following this line we construct a simple framework to study the physics of nuclear direct reactions with heavy ions, and in particular to understand how the continuum impacts on the reaction. To better focus on our aim, our model is kept as simple as possible: we consider "semiclassical" motion, and reduce the problem to one dimension. This allows us to concentrate on the study of the role of the continuum in a more systematic and generalized way (varying the mean field properties of the colliding nuclei and the quantum representation of the continuum), and to test $\mathrm{CC}$ techniques directly comparing the results to the solution of the time-dependent Schrödinger equation.

So, we consider the problem of one particle [21], initially bound in a one-dimensional potential well (the "target" $T$ ), subject to an external field, which is also represented by a potential well (the "projectile" $P$ ), and which moves in one dimension according to a predetermined classical equation of motion. This situation describes schematically the collision between two nuclei, each one modeled by a potential well, and it permits us to understand the mechanisms which govern a nuclear reaction by following the evolution of the wave function associated to the particle. Initially, since we assume the particle to be a valence nucleon of the target, it coincides with an eigenstate of the target potential well. During the reaction the particle will feel the interaction with the incident nucleus, i.e., the projectile potential well. Depending on the parameters characterizing the reaction, the particle will more likely remain in its initial state (elastic scattering), jump to an excited bound level of the target (inelastic scattering), be transferred to a bound level of the other nucleus, or it could leave the initial nucleus and escape to the continuum (breakup). As a consequence, the particle wave function will change, according to the probability to excite the different reaction channels.

This paper is structured as follows: in Sec. II we detail the model for the numerical "exact" solution (Sec. II A) as well as the coupled-channels description (Sec. II B). In particular, in Sec. II B, we discuss the treatment of the continuum in our $\mathrm{CC}$ calculation. In Sec. III we apply our models to different dynamical situations; finally we summarize our results and give our conclusions in Sec. IV.

\section{THE MODEL}

Let us start the model description by specifying the Hamiltonian and the initial conditions. The system is described with the Hamiltonian

$$
\mathcal{H}(x, t)=-\frac{\hbar^{2}}{2 \mu} \frac{\partial^{2}}{\partial x^{2}}+V_{T}(x)+V_{P}\left(x-x_{p}(t)\right),
$$

where we include two potentials $V_{T}$ and $V_{P}$, chosen with a Woods-Saxons shape, and associated to target and to projectile, respectively, and $\mu$ represents the reduced mass of the valence particle. In our semiclassical model the target is at rest and only the projectile potential well moves according to a classical trajectory. We use the trajectory proposed in Ref. [22]:

$$
x_{p}(t)=x_{0}+\sqrt{\rho^{2}+(v t)^{2}}-\rho,
$$

that accounts for the projectile motion with fixed asymptotic velocity $v$ at large distances, corresponding to an asymptotic energy $E_{P}=1 / 2 m v^{2}$, which determines the effective duration of the reaction (the higher the asymptotic energy, the faster is the reaction), and a distance of closest approach $x_{0}$ between the two centers of the potentials. This trajectory allows the projectile to change its acceleration over the distance $\rho$ (in our model of the order of $2 \mathrm{fm}$ ), thus simulating the nuclear interaction with the target; at $t= \pm \infty$ the trajectory tends to a uniform motion with zero acceleration. The turning point of the collision corresponds to $t=0 \mathrm{ps}$. Each process is divided in $\tau$ steps, so the total duration is $\tau d t$. We determine the time interval as $d t=d x / 4 v$, small enough to provide a stable solution for a spatial grid step length of $d x=0.2 \mathrm{fm}$ at maximum and the asymptotic energies studied in the present paper.

Upon variation of these parameters, one can simulate different kinematical conditions due to different bombarding energies and impact parameters (corresponding to partial waves in a quantum-mechanical description), while the choice of the parameters of the two potentials accounts for the different masses of the colliding nuclei, the $Q$ values of the different final channels, as well as the possibility of simulating weak binding conditions.

By solving separately the time-independent Schrödinger equation for each well $J=(T, P)$

$$
\mathcal{H}_{J} \Phi^{J}(x)=E_{J} \Phi^{J}(x),
$$

with the Hamiltonian

$$
\mathcal{H}_{J}=-\frac{\hbar^{2}}{2 \mu} \frac{d^{2}}{d x^{2}}+V_{J}(x),
$$

we obtain two sets of bound levels $\Phi^{J}(x)$ associated to negative eigenvalues [23] $\left(E_{J}<0\right)$, and a continuum associated to positive energies $\left(E_{J}>0\right)$. By applying the discretization methods detailed in Ref. [24], we can also define a set of square-integrable functions associated to a 
finite number of positive energies, the so-called discretized continuum pseudostates. Each wave function is normalized as $\int|\Phi(x)|^{2} d x=1$.

Depending on the kind of reaction under study we will select one of the bound levels as the initial state of our singleparticle wave function. For example, to describe a pick-up reaction we will choose as initial state a single-particle level in the target. In the simulations presented here we will always choose a target wave function as the initial one.

The problem can be solved in many different ways. Two such methods are considered and compared here. On one hand, we consider the exact solution of the problem by numerically solving the corresponding time-dependent Schrödinger equation (Sec. II A). On the other hand, we consider an approximate solution using the coupled-channels method, which is usually applied in solving three-dimensional scattering problems (Sec. II B). From this comparison, we expect to get further insight on the accuracy and limitations of the coupledchannels approach to quantum collisions.

\section{A. Exact time-dependent solution}

In the case of the exact solution, we proceed to compute the time evolution of the valence neutron wave function $\Psi(x, t)$ by numerically solving the time-dependent Schrödinger equation

$$
i \hbar \frac{\partial}{\partial t} \Psi(x, t)=\mathcal{H}(x, t) \Psi(x, t),
$$

with the Hamiltonian (1).

The wave function $\Psi$ is confined within an interval containing the two wells. It is calculated at fixed points with coordinates $x_{j}$ separated by $d x$. According to Ref. [25], one can solve the problem by using a finite-difference approximation of the Hamiltonian, giving rise to the following tridiagonal form:

$$
\begin{aligned}
\mathcal{H}_{j k}= & -\frac{\hbar^{2}}{2 \mu d x^{2}}\left(\delta_{j k+1}+\delta_{j k-1}-2 \delta_{j k}\right) \\
& +\delta_{j k}\left[V_{T}\left(x_{j}\right)+V_{P}\left(x_{j}-x_{p}(t)\right)\right],
\end{aligned}
$$

and the time evolution of the wave function is governed by the so-called Padé approximation of the evolution operator:

$$
\Psi(t+d t)=\left(1+\frac{i d t}{2 \hbar} \mathcal{H}\right)^{-1}\left(1-\frac{i d t}{2 \hbar} \mathcal{H}\right) \Psi(t),
$$

where $d t$ is a finite time step, and $\mathcal{H}$ is the matrix in Eq. (6) at the intermediate time $t+d t / 2$. Note that the evolution operator is a unitary operator.

An alternative approach is to integrate the differential equation using a finite-difference method, such as the Runge-Kutta method. For that, in this paper we make use of the routines D02PVF and D02PCF of the NAG library [26]. Although this solution prevents us from a complete control of the code, it was found to be faster than the Padé method. We have also verified that both methods lead to identical results.

At the end of the time evolution, when $t=t_{f}$, we can compute the final probabilities for each reaction channel by projecting the final wave function $\Psi\left(x, t_{f}\right)$ onto the corresponding $i$ th eigenstates of each well obtained by solving
Eq. (4) for each potential (target bound states $J=T$ for elastic and inelastic, projectile bound states $J=P$ for transfer channels):

$$
\begin{aligned}
\mathcal{P}_{\text {elastic }} & =\left|\left\langle\Phi_{i=\text { g.s. }}^{T}(x) \mid \Psi\left(x, t_{f}\right)\right\rangle\right|^{2}, \\
\mathcal{P}_{\text {inelastic }} & =\left|\left\langle\Phi_{i \neq \text { g.s. }}^{T}(x) \mid \Psi\left(x, t_{f}\right)\right\rangle\right|^{2}, \\
\mathcal{P}_{\text {transfer }} & =\left|\left\langle\Phi_{i}^{P}\left(x-x_{p}\left(t_{f}\right)\right) \mid \Psi\left(x, t_{f}\right)\right\rangle\right|^{2} .
\end{aligned}
$$

We can also evaluate the breakup probability either by direct subtraction,

$$
\mathcal{P}_{\text {breakup }}=1-\mathcal{P}_{\text {elastic }}-\mathcal{P}_{\text {inelastic }}-\mathcal{P}_{\text {transfer }},
$$

or by projecting the final wave function onto a complete set of continuum states $\varphi(k, x)$ depending on the asymptotic wave number $k= \pm \sqrt{\frac{2 \mu E}{\hbar^{2}}}$ :

$$
\mathcal{P}_{\text {breakup }}=\int d E \sqrt{\frac{\mu}{2 E \hbar^{2}}} \mathcal{P}(k)=\int d k\left|\left\langle\varphi(k, x) \mid \Psi\left(x, t_{f}\right)\right\rangle\right|^{2} .
$$

In one dimension, for each positive energy there are two degenerate continuum wave functions with momentum $k$, one incoming from the left and the other from the right. For each energy we consider the symmetric and antisymmetric combinations of the momentum normalized continuum wave functions $[24,27]$.

\section{B. Approximate solution within the coupled-channels method}

The same problem can be solved with the so-called coupled-channels method, which is a popular framework used to describe quantum collision problems in atomic, molecular, and nuclear physics. For this calculation we follow the formulation of Ref. [22], and we take into account two finite sets of wave functions, related to the target and the projectile potentials: $\Phi_{j}^{T}(x)$ and $\Phi_{j}^{P}(x)$, of $N_{T}$ and $N_{P}$ states, respectively. For collisions among tightly bound systems, the basis expansion is usually restricted to bound states of the projectile and target systems. However, when one of them is weakly bound, it is important to include also continuum states. For that, it is convenient to use a discrete representation of square-integrable functions, as those discussed in Ref. [24] (we will discuss in more detail the inclusion of the continuum in coupled-channels method in Sec. II B 1). Moreover, they are defined in a one-dimensional spatial grid, the origin of which corresponds to the center of the target potential, which also corresponds to the laboratory frame. A different choice, like the center-of-mass frame of reference in which the two potentials are moving, would have implied a careful treatment of target and projectile wave functions due to the noncovariance of the Schrödinger equation [19,27]. In addition, these two bases are nonorthogonal so we will solve this problem introducing the dual bases $\omega_{j}^{J}(x, t)$, as explained in Refs. [22,27-30]. They are, respectively, associated to each well and conjugated to the channel wave functions of each potential, through the definition

$$
\left\langle\Phi_{m}^{I} \mid \omega_{n}^{J}\right\rangle=\delta_{I, J} \delta_{n, m},
$$

where $I, J=T, P$ and $n, m=1,2, \ldots, N_{(T, P)}$. 
In the CC approach, the wave function describing the entire system is expressed as a combination of target and projectile states:

$$
\Psi(x, t)=\sum_{j=1}^{N_{T}} c_{j}^{T}(t) \Phi_{j}^{T}(x)+\sum_{j=1}^{N_{P}} c_{j}^{P}(t) \Phi_{j}^{P}\left(x-x_{p}(t)\right),
$$

and the solution of the problem is reduced to the determination of the time evolution of the coefficients $c_{j}^{J}(t)$ from the finite set of coupled differential equations:

$$
\begin{aligned}
& i \hbar \frac{d c_{j}^{T}(t)}{d t}=\sum c_{k}^{T}(t)\left\langle\omega_{j}^{T}\left|V^{P}\right| \Phi_{k}^{T}\right\rangle+\sum c_{k}^{P}(t)\left\langle\omega_{j}^{T}\left|V^{T}\right| \Phi_{k}^{P}\right\rangle, \\
& i \hbar \frac{d c_{j}^{P}(t)}{d t}=\sum c_{k}^{T}(t)\left\langle\omega_{j}^{P}\left|V^{P}\right| \Phi_{k}^{T}\right\rangle+\sum c_{k}^{P}(t)\left\langle\omega_{j}^{P}\left|V^{T}\right| \Phi_{k}^{P}\right\rangle,
\end{aligned}
$$

where the bases functions and potentials related to $T$ and $P$ depend on $(x)$ and $\left(x-x_{p}(t)\right)$, respectively. These equations are solved with the initial conditions $c_{j}^{P}(t=-\infty)=0$ and $c_{j}^{T}(t=-\infty)=\delta_{i, j}$, where $i$ indicates one of the bound states in the target potential well.

To derive Eqs. (15), we first insert Eq. (14) of the total wave function $\Psi(x, t)$ into the time-dependent Schrödinger Eq. (5), thus obtaining

$$
\begin{aligned}
& \sum_{j} i \hbar \frac{d c_{j}^{T}(t)}{d t} \Phi_{j}^{T}(x)+\sum_{j} i \hbar \frac{d c_{j}^{P}(t)}{d t} \Phi_{j}^{P}\left(x-x_{p}(t)\right) \\
& =\sum_{j} c_{j}^{T}\left[\mathcal{H}(x, t)-\mathcal{H}_{T}(x)\right] \Phi_{j}^{T}(x) \\
& \quad+\sum_{j} c_{j}^{P}\left[\mathcal{H}(x, t)-\mathcal{H}_{P}\left(x-x_{p}(t)\right)\right] \Phi_{j}^{P}\left(x-x_{p}(t)\right)
\end{aligned}
$$

where $\mathcal{H}_{J}$ is the Hamiltonian corresponding to the potential well $J=T, P$ of Eq. (4), and $\mathcal{H}$ is the Hamiltonian of the full system presented in Eq. (1).

In problems involving different mass partitions, one may use the so-called prior and post representations of the Hamiltonian, depending on whether one considers the projectile-target combination of the initial or final states; by definition they have to give the same results. Equations (14) and (15) are constructed in prior representation. In post representation we can expand the system wave function on the dual basis:

$$
\Psi(x, t)=\sum_{n} \tilde{c}_{n}^{T}(t) \omega_{n}^{T}(x)+\sum_{n} \tilde{c}_{n}^{P}(t) \omega_{n}^{P}\left(x-x_{p}(t)\right),
$$

and, following a derivation similar to the one given for the prior representation, we obtain the corresponding set of coupled equations:

$$
\begin{aligned}
& i \hbar \frac{d \tilde{c}_{n}^{T}(t)}{d t}=\sum \tilde{c}_{m}^{T}(t)\left\langle\Phi_{n}^{T}\left|V^{P}\right| \omega_{m}^{T}\right\rangle+\sum \tilde{c}_{m}^{P}(t)\left\langle\Phi_{n}^{T}\left|V^{P}\right| \omega_{m}^{P}\right\rangle, \\
& i \hbar \frac{d \tilde{c}_{n}^{P}(t)}{d t}=\sum \tilde{c}_{m}^{T}(t)\left\langle\Phi_{n}^{P}\left|V^{T}\right| \omega_{m}^{T}\right\rangle+\sum \tilde{c}_{m}^{P}(t)\left\langle\Phi_{n}^{P}\left|V^{T}\right| \omega_{m}^{P}\right\rangle,
\end{aligned}
$$

where the bases functions and potentials related to $T$ and $P$ depend on $(x)$ and $\left(x-x_{p}(t)\right)$, respectively. From the total wave function $\Psi(x, t)$, we can also extract amplitudes for excitation and transfer in post and prior representations through the expressions

$$
\begin{aligned}
& \tilde{c}_{n}^{I}(t)=\left\langle\omega_{n}^{I} \mid \Psi\right\rangle, \\
& c_{n}^{I}(t)=\left\langle\Phi_{n}^{I} \mid \Psi\right\rangle .
\end{aligned}
$$

Due to post-prior symmetry, the amplitudes in the two representations are related by

$$
\begin{gathered}
\tilde{c}_{n}^{T}(t)=c_{n}^{T}(t)+\sum_{m}\left\langle\Phi_{n}^{T} \mid \Phi_{m}^{P}\right\rangle c_{m}^{P}(t), \\
\tilde{c}_{n}^{P}(t)=c_{n}^{P}(t)+\sum_{m}\left\langle\Phi_{n}^{P} \mid \Phi_{m}^{T}\right\rangle c_{m}^{T}(t) .
\end{gathered}
$$

The probabilities to populate the different final channels are defined as

$$
\mathcal{P}_{j}^{J}=\left|c_{j}^{J}\left(t_{f}\right)\right|^{2}
$$

in the prior representation, or as

$$
\tilde{\mathcal{P}}_{j}^{J}=\left|\tilde{c}_{j}^{J}\left(t_{f}\right)\right|^{2}
$$

in the post representation. The index $j$ denotes a label to the quantum number of the final state in one of the two wells.

Because of the nonorthogonality of the basis states, the sum of these "probabilities" is not conserved during the collision. If we instead define the probabilities by

$$
\mathcal{P}_{j}^{J}=\operatorname{Re}\left\{\left[c_{j}^{J}\left(t_{f}\right)\right]^{*} \tilde{c}_{j}^{J}\left(t_{f}\right)\right\},
$$

conservation of total probability is always fulfilled within the coupled-channel formalism. This follows from the fact that the matrix governing the time evolution of the amplitudes in the post representation is minus the Hermitian conjugate of the matrix that determines the time evolution of the amplitudes in the prior representation. We shall therefore call Eq. (23) the unitary representation of probabilities. However, there is no guarantee that these quantities are always non-negative during the collision.

After the collision, when all overlaps between the basis states in the two wells vanish, the amplitudes for a given transition are the same in the post and prior representation, as evident from Eq. (20). This so-called post-prior symmetry implies that the total probability is conserved once the collision is over, also in a truncated coupled-channel treatment.

\section{Inclusion of the continuum in the coupled-channels method}

In Ref. [22] only bound states were included in the bases and, hence, breakup channels were omitted. This is possibly justified for tightly bound systems, but not for weakly bound ones, for which the coupling to these channels can be very important.

In a coupled-channels scheme one cannot include the full continuum spectrum, since these states form a continuum of energies. Moreover, the fact that these states are not square integrable poses numerical problems since the coupling potentials become of infinite range. To overcome these difficulties, 
it is customary to resort to an approximate, discrete description of the continuum. To describe the breakup process in CC a set of discretized continuum states defined on a finite spatial range is usually included for one or more subsystems of the reaction constituents. Even if the description of the process is supposed to occur at the end of the reaction, when the distance between the constituents tends to infinity, this constitutes a nonphysical situation. In fact, a proper description of the continuum, even if discretized, should take into account all the fragments involved in the reaction. This is what is done in the Faddeev formalism [31].

In time-dependent approaches, like the one we apply, to include a proper description of the continuum is a challenge. Here, we use the pseudostates introduced in Ref. [24]; in particular, we discretize the continuum in an infinite squarewell basis (BOX method in Ref. [24]). We apply the same approach as in CDCC and provide discretized continuum pseudostates for each potential well. To include them in our coupled-channels calculation, we should ensure that at the end of the time evolution there is no overlap between target and projectile bases. This is due to the fact that the two bases are not mutually orthogonal, so while they overlap the problem loses unitarity, as we mentioned in Sec. II B. After the reaction, when the projectile is far enough for these overlaps to vanish, the problem has restored its unitarity and we can evaluate the final probabilities. Thus, if we include the continuum we need to restrict it to a small range $\left[-x_{b}^{J},+x_{b}^{J}\right]$ centered in the corresponding potential $J=T, P$, in order that $x_{b}^{T}+x_{b}^{P}>$ $x_{p}\left(t_{f}\right)$. A limitation of this method is that the choice of $x_{b}^{J}$ is done a posteriori to include the breakup component into the continuum interval. In situations in which the breakup channel is strongly dominant, it could become impossible to apply this method because the continuum component of the wave function might not be localized close to one of the potential wells. By comparing our $\mathrm{CC}$ results to the exact solution of the Schrödinger equation in this simple one-dimensional framework, we are able to understand the limitation of this description of the continuum.

\section{NUMERICAL RESULTS: COMPARISON OF EXACT AND APPROXIMATE METHODS}

In previous works we have applied the present approach on a variety of model cases to study different aspects of the direct reaction mechanism, always considering bound and weakly bound systems. In particular, we have tested the model by varying the distance of closest approach between the two potential wells and the $Q$ value of the reaction in Refs. [27,32,33], and we have studied the role of the continuum in different configurations in Refs. [27,34,35]. To explore different kinematical situations, we have performed further calculations. In each case, we present the exact timedependent solution (Sec. II A) and compare this result with the approximate solutions obtained with the coupled-channels calculation presented, as described in Sec. II B. The chosen model cases have been selected to illustrate several physical situations in which different reaction channels are favored. For each case we have highlighted an analogous problem solved in a three-dimensional CDCC environment, discussing the
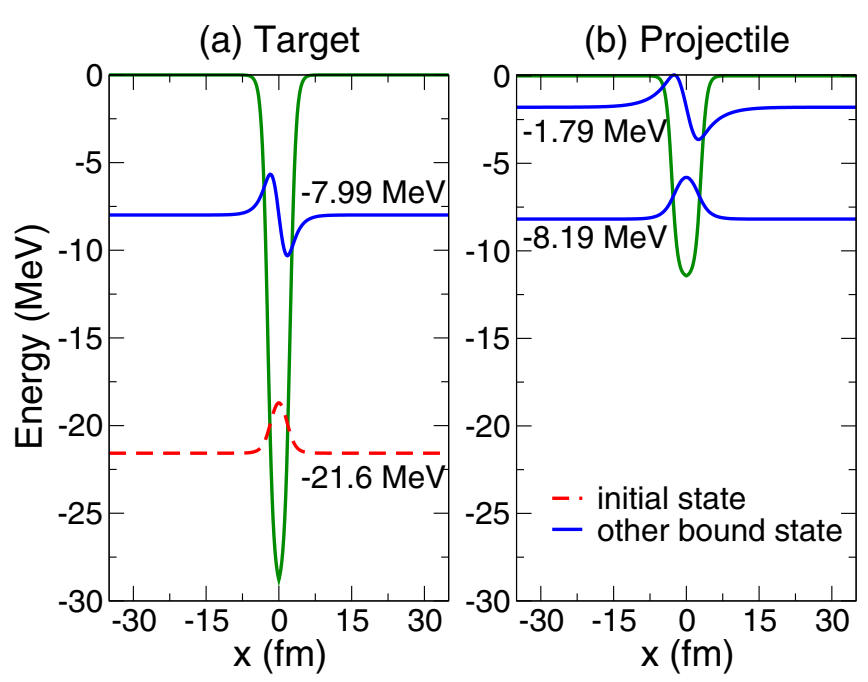

FIG. 1. Target (a) and projectile (b) Woods-Saxon potentials for the case A. Their bound states are schematically depicted in correspondence with their binding energy. The initial state, in this case the target ground state, is the dashed red curve.

main challenges experienced in the $3 \mathrm{D}$ situation within our simplified framework.

\section{A. Case A}

We start with the simplest case of a well-bound target in which we observe a dominance of elastic and inelastic channels. The target and projectile potentials are depicted in Fig. 1 with their respective bound-state wave functions. The lowest state in the target is chosen as initial state for this reaction; its wave function is shown as a dashed red curve in Fig. 1. The projectile follows the trajectory (2) with an asymptotic velocity of $0.1 \times c$, which corresponds to an asymptotic energy of $5.0 \mathrm{MeV}$. The reduced mass for this system is $1.001 \mathrm{amu}$; the time evolution is divided in $\tau=2510$ steps.

In Fig. 2 we present the exact wave-function squared value at different moments of the time evolution. One can see that a small component of the system wave function is transferred and remains bound to the projectile potential well as it moves away after the collision.

In Table I we present the results obtained by solving the problem using different approaches and different bases.

In particular, they correspond to the following, as shown in Table I.

TABLE I. Final probabilities for the model case A obtained with the exact and the coupled-channels methods.

\begin{tabular}{lrcccr}
\hline \hline & Exact & $\mathrm{CC}^{(1)}$ & $\mathrm{CC}^{(2)}$ & $\mathrm{CC}^{(3)}$ & $\mathrm{CC}^{(4)}$ \\
\hline Elastic & $86.1 \%$ & $89.8 \%$ & $89.4 \%$ & $74.1 \%$ & $84 \%$ \\
Inelastic & $9.8 \%$ & $10.1 \%$ & $10.3 \%$ & $18.4 \%$ & $11.6 \%$ \\
Transfer g.s. & $2.5 \%$ & & & $6.8 \%$ & $2.3 \%$ \\
Transfer 1 & $0.8 \%$ & & & $0.56 \%$ & $1.9 \%$ \\
Breakup & $0.7 \%$ & & $0.3 \%$ & & $0.13 \%$ \\
\hline \hline
\end{tabular}




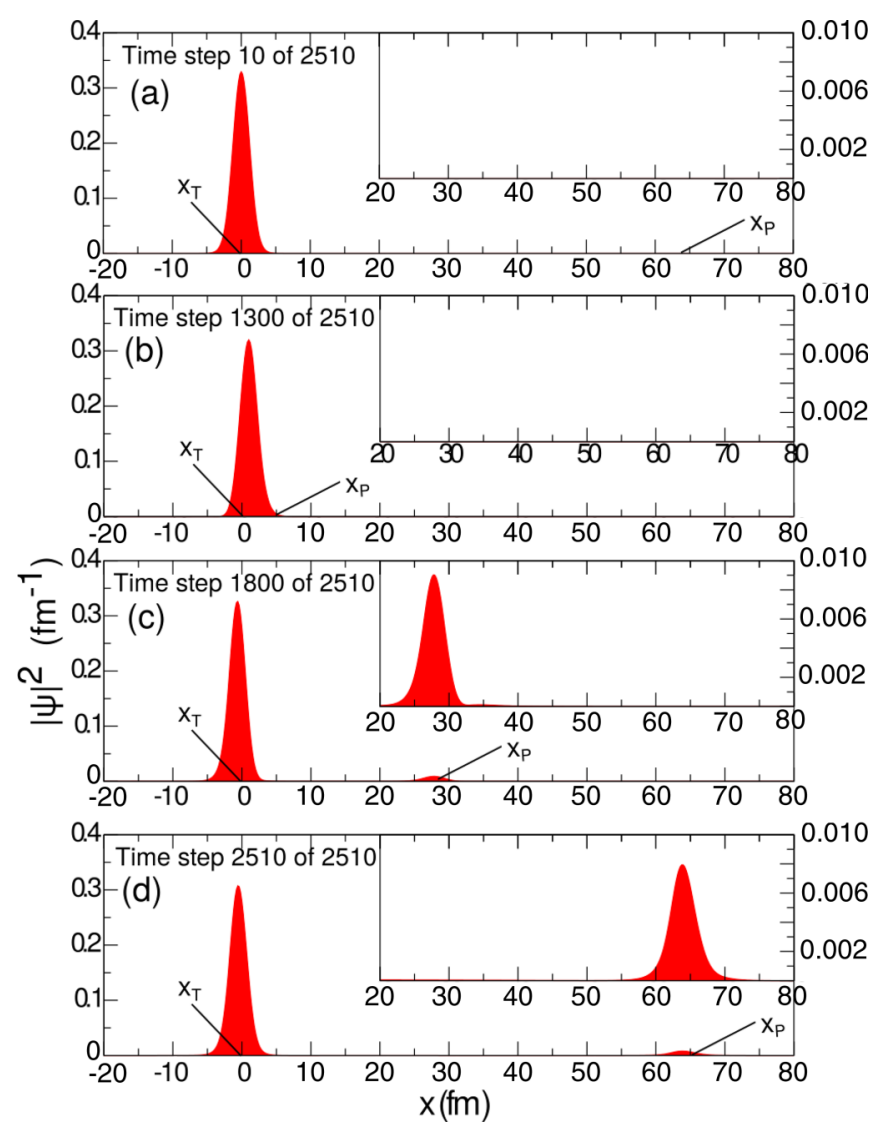

FIG. 2. Model case A, with the exact squared total wave function at different times and a zoom for $x>20 \mathrm{fm}$ in the inset. Target and projectile potential wells position is indicated by the labels $x_{T}$ and $x_{P}$, respectively. The elapsed time for each frame is also indicated: this process is composed by 2510 time steps; the turning point $t=0$ ps occurs at step 1255 .

(1) Exact: Calculation done by solving numerically the time-dependent Schrödinger Eq. (5). The different results represent the probability for the valence neutron to remain in the ground state (elastic) or to directly excite the corresponding bound states of the two potential wells (inelastic and transfer) or to excite continuum states (breakup).

(2) $\mathrm{CC}^{(1)}$ : Coupled-channels calculation in which only the two target bound states are included.

(3) $\mathrm{CC}^{(2)}$ : Coupled-channels calculation using target bound levels plus the first ten continuum pseudostates obtained in a range equal to the maximum radius of the grid.

(4) $\mathrm{CC}^{(3)}$ : Coupled-channels calculation including only target and projectile bound states.

(5) $\mathrm{CC}^{(4)}$ : Coupled-channels calculation including target and projectile bases composed by bound and five continuum pseudostates calculated in a $[-20 ; 20]-\mathrm{fm}$ range centered in the respective potential. It corresponds to an energy cutoff in the continuum of $4 \mathrm{MeV}$.

By comparing the results of Table I, we note that the coupled-channels calculation without transfer and continuum channels, i.e., $\mathrm{CC}^{(1)}$, reproduces rather well the elastic and inelastic probabilities. This is a consequence of the dominance of the elastic channel and, to a lesser extent, the inelastic

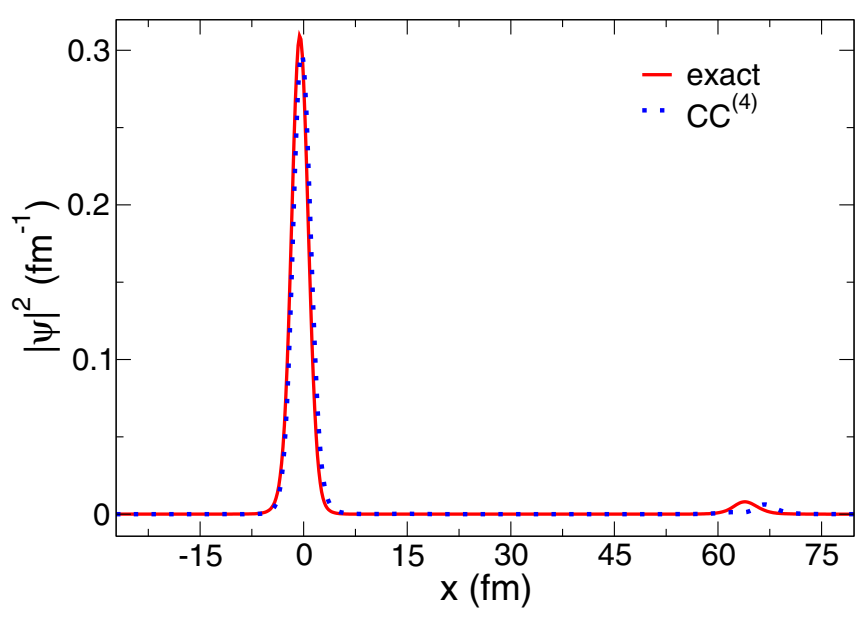

FIG. 3. Final exact wave function (solid red line) and final $\mathrm{CC}^{(4)}$ wave function (dotted blue line). The projectile potential is placed at $x_{P}\left(t_{f}\right)=65 \mathrm{fm}$.

channel in this case. Including the breakup $\left(\mathrm{CC}^{(2)}\right)$ channel alone does not improve the result, because it is not possible to describe the transfer in this configuration. Interestingly, a calculation with elastic, inelastic, and transfer channels $\left(\mathrm{CC}^{(3)}\right)$ is not enough to reproduce the expected values, even if those are the dominant channels in this case. By including all possible channels simultaneously $\left(\mathrm{CC}^{(4)}\right)$ we obtain a fine agreement with the numerical solution.

In Fig. 3, we compare the final exact wave function (solid red line), already reported in the upper panel of Fig. 2(d), to the wave function obtained from calculation $\mathrm{CC}^{(4)}$ using the final coupled-channels coefficients in Eq. (14) (dotted blue line). We observe a quite good agreement between the two results, the slight differences being due to the overestimation of the excitation of the first excited states of both target and projectile wells by the $\mathrm{CC}$ calculation. To further understand the role of continuum states in this process, we construct the exact momentum-normalized bases $\varphi(k, x)$ for the two potential wells. We then calculate the probability as a function of the momentum $\mathcal{P}(k)$ following Eq. (12). The results are displayed in Fig. 4: the exact (solid red) and $\mathrm{CC}^{(4)}$ (dashed blue) final wave functions from Fig. 3 have been projected onto the target and projectile exact continuum in the upper and lower panels, respectively. We can notice how the "projectile continuum" plays a more relevant role than the "target continuum", and also how well the $\mathrm{CC}^{(4)}$ calculation works in this case. For the target continuum we observe that the coupled-channels calculation does not reproduce accurately the structure observed at lower momenta. Anyway, as already observed, the total strength is negligible with respect to the role of the other well. It should be noticed that the incident energy of $5 \mathrm{MeV}$ corresponds to a threshold of $k=0.49 \mathrm{fm}^{-1}$; the excitation slightly exceeds this limit probably due to the nonconservation of energy in semiclassical descriptions [36].

This case is ideally described within the CC method, given the dominance of the elastic channel. Many authors have found an excellent agreement with elastic and transfer experimental cross sections, provided that breakup channels 

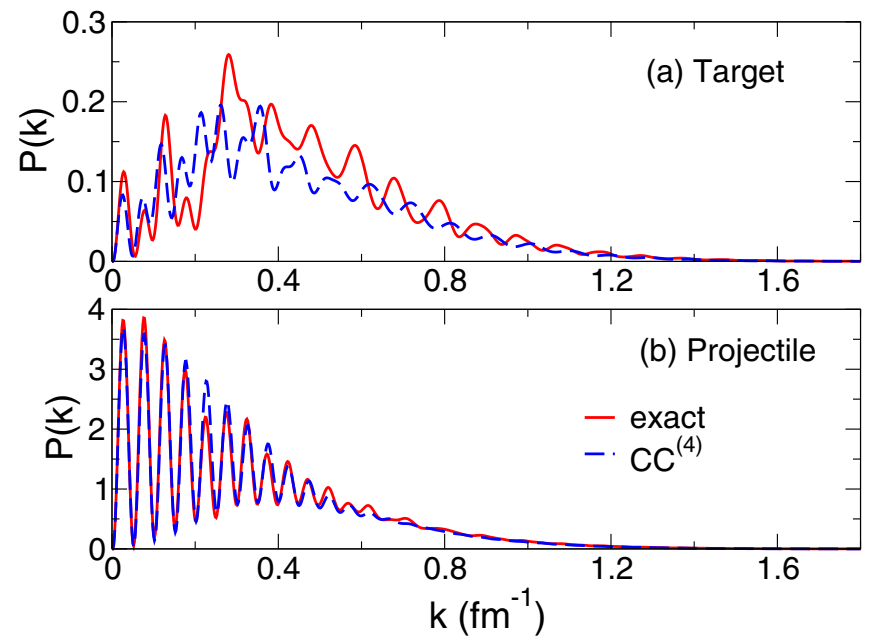

FIG. 4. Breakup probabilities as a function of the momentum in the continuum. The upper and lower panels correspond to the projection on target and projectile states, respectively. Solid red lines indicate the exact final wave function, while dashed blue lines indicate the $\mathrm{CC}^{(4)}$ final one.

are properly included in the CDCC calculation (see, e.g., Refs. [13,37-39]). Also, some authors have suggested that continuum-continuum couplings have a strong influence in the complete and incomplete fusion reaction for weakly bound nuclei [40-42]. The present model case confirms the crucial role of the continuum, and contributes to demonstrate that even if the system under study is well bound and the breakup component is negligible, the inclusion of continuum states can be essential to get a proper description of the reaction. In this situation in which the breakup component is not dominant, to include two sets of continuum states, each one related to one potential well, allows us to obtain a result in agreement with the expected exact one. So, this treatment of the continuum could constitute an acceptable approximation in these conditions.

\section{B. Case B}

In this second case the initial target state is extremely weakly bound and consequently the breakup channel is the most relevant. The target and projectile potentials are depicted in Fig. 5 with their respective bound-state wave functions. The initial state is the dashed red curve corresponding to the target bound state. The energy of this state is $-0.276 \mathrm{MeV}$ and its weakly bound nature is clearly evident from the extended tail of its wave function. The projectile follows the trajectory (2) with an asymptotic velocity of $0.1 \times c$ corresponding to an incident energy of $5.0 \mathrm{MeV}$, and with a reduced mass of 1.001 amu; the time evolution is divided in $\tau=4790$ steps.

The evolution of the exact wave function during the collision is presented in Fig. 6. In this case, both the transfer and the continuum components of the system wave function are clearly evident.

The results for case B, obtained applying different methods, are presented in Table II. In particular, they correspond to the following, as shown in Table II.
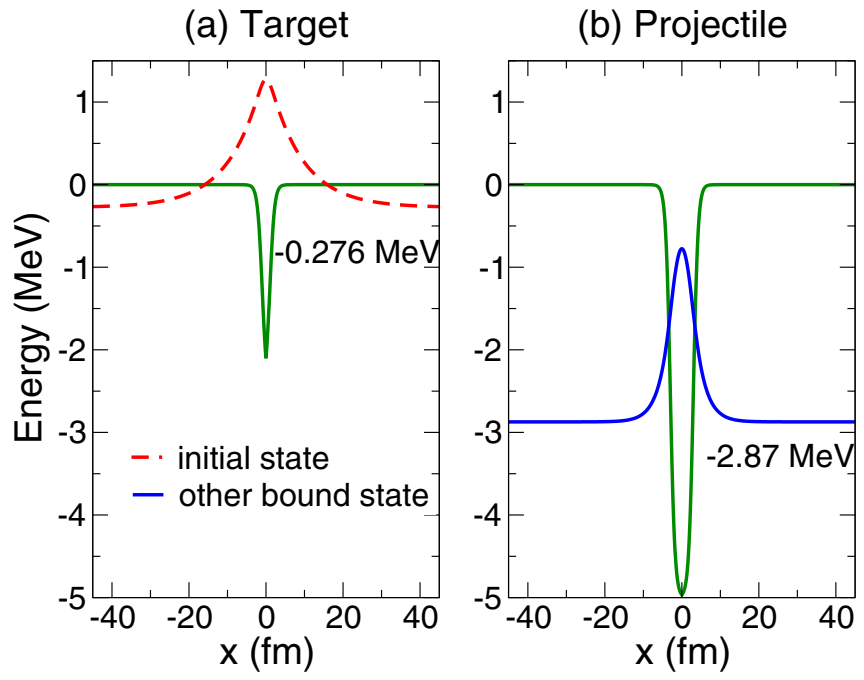

FIG. 5. Target (a) and projectile (b) Woods-Saxon potentials for the case B. Their bound states are schematically depicted in correspondence with their binding energy. The initial state, in this case the target ground state, is the dashed red curve.
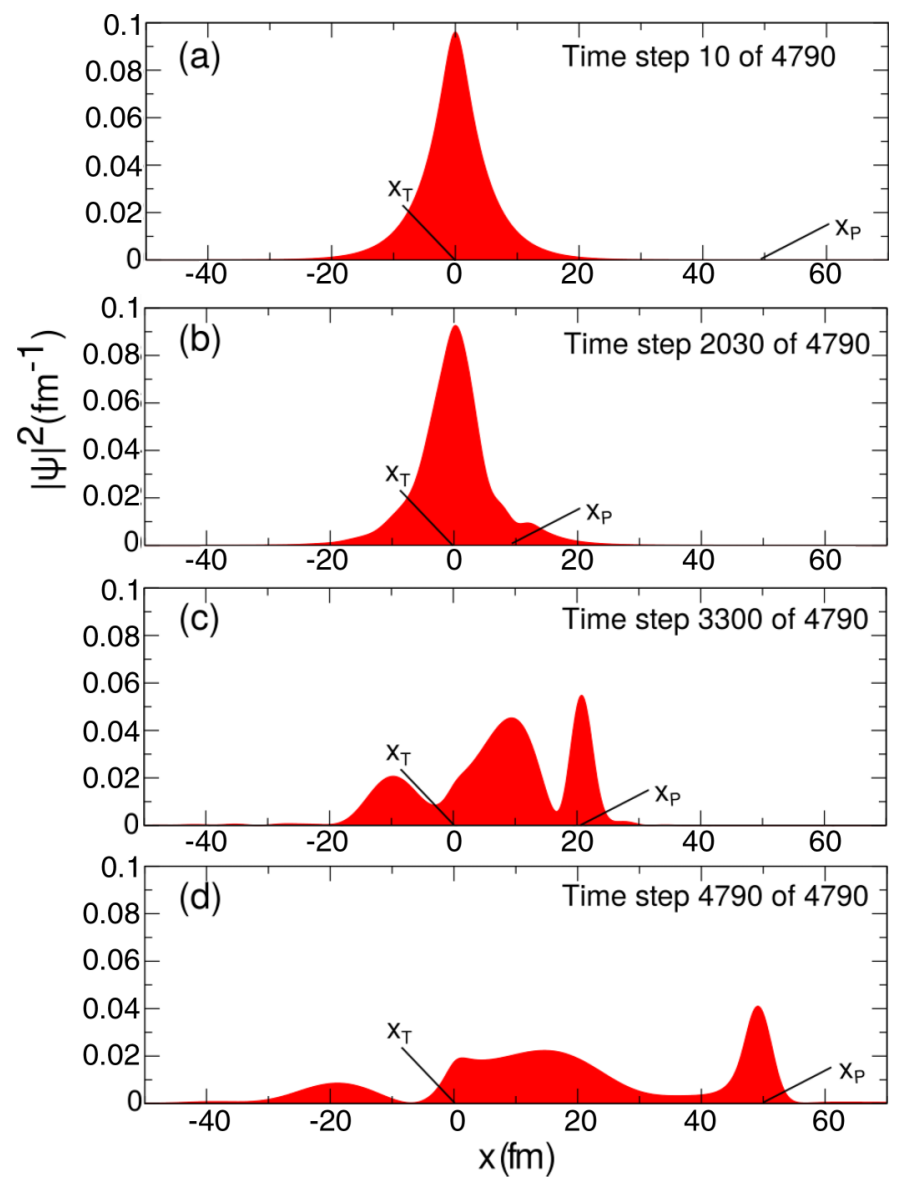

FIG. 6. Model case B, with the exact squared total wave function at different times. Target and projectile potential wells position is indicated by the labels $x_{T}$ and $x_{P}$, respectively. The elapsed time for each frame is also indicated: this process is composed by 4790 time steps; the turning point $t=0 \mathrm{ps}$ occurs at step 2395 . 
TABLE II. Final probabilities for the model case B obtained with the exact and the coupled-channels methods.

\begin{tabular}{lrccc}
\hline \hline & Exact & $\mathrm{CC}^{(1)}$ & $\mathrm{CC}^{(2)}$ & $\mathrm{CC}^{(3)}$ \\
\hline Elastic & $20.94 \%$ & $22.9 \%$ & $21.9 \%$ & $25.8 \%$ \\
Transfer & $6.73 \%$ & & $<10^{-3} \%$ & $0.3 \%$ \\
Breakup & $72.32 \%$ & $77.1 \%$ & $78.2 \%$ & $73 \%$ \\
\hline \hline
\end{tabular}

(1) $\mathrm{CC}^{(1)}$ : Coupled-channels calculation in which the target bound state and ten target continuum states within the $[-40 ; 40]-\mathrm{fm}$ range are included.

(2) $\mathrm{CC}^{(2)}$ : Coupled-channels calculation using target and projectile bound levels plus 50 continuum target pseudostates obtained in a radius equal to $40 \mathrm{fm}$.

(3) $\mathrm{CC}^{(3)}$ : Coupled-channels calculation including target and projectile bases composed by bound and five continuum pseudostates calculated in a $[-22 ; 22]-f m$ range centered in the respective potential. It corresponds to an energy cutoff in the continuum of about $4 \mathrm{MeV}$.

Including only target basis states, calculation $\mathrm{CC}^{(1)}$ cannot describe alone the three channels of this process, even if it reproduces quite well the proportion between elastic and breakup channels. By adding the projectile bound state $\left(\mathrm{CC}^{(2)}\right)$ the elastic channel is better reproduced, but the transfer is not well accounted for. Including also the projectile continuum improves the result $\left(\mathrm{CC}^{(3)}\right)$, thus showing that in a perturbation picture the transfer is not a one-step process, but it is reached through successive steps involving the continuum. Multistep processes in direct reactions are traditionally inferred by CDCC calculations, and, as we have seen in the previous section, continuum-continuum couplings play a crucial role $[41,43,44]$.

In Fig. 7 we show the final wave functions for the exact (solid red), $\mathrm{CC}^{(1)}$ (dashed magenta), and $\mathrm{CC}^{(3)}$ (dotted blue) calculations. Neither of the two $\mathrm{CC}$ wave functions reproduces the shape of the final exact wave function, al-

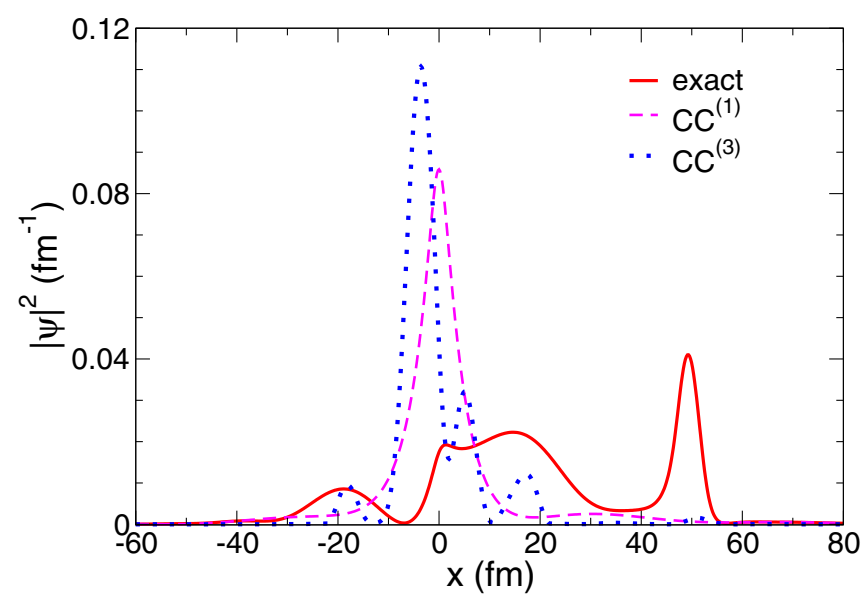

FIG. 7. Final exact wave function (solid red line), final $\mathrm{CC}^{(1)}$ wave function (dashed magenta line), and final $\mathrm{CC}^{(3)}$ wave function (dotted blue line). The projectile potential is placed at $x_{P}\left(t_{f}\right)=$ $50 \mathrm{fm}$.
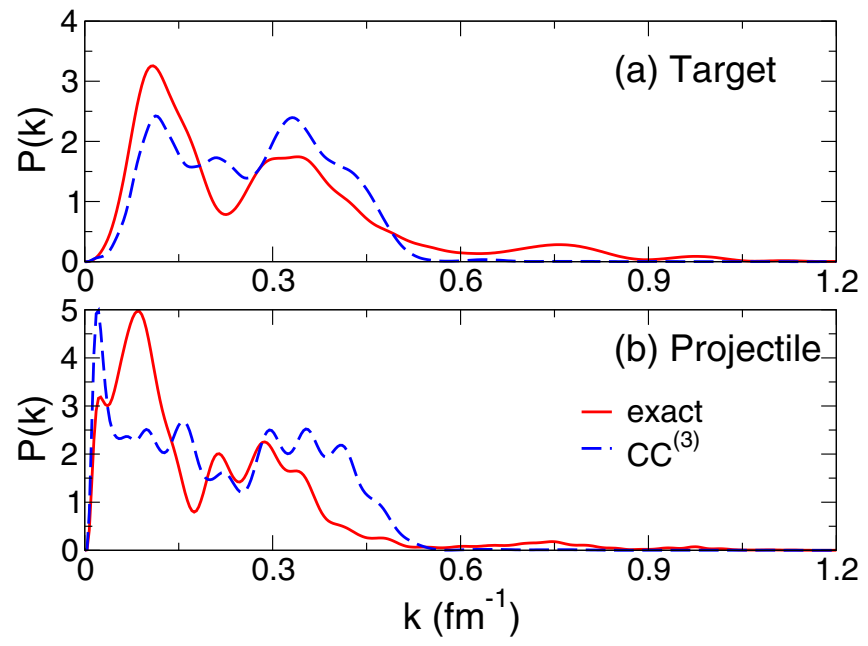

FIG. 8. Breakup probabilities as a function of the momentum in the continuum. The upper and lower panels correspond to the projection on target and projectile states, respectively. Solid red lines indicate the exact final wave function, while dashed blue lines indicate the $\mathrm{CC}^{(3)}$ final one.

though their breakup probabilities are in agreement. This is partly explained by the failure in describing the transfer component, and, more importantly, by the spatial restriction of the pseudostates. This is also seen in Fig. 8, where we show the projection of the exact (solid red) and $\mathrm{CC}^{(3)}$ (dashed blue) wave functions onto the exact continuum $\varphi(k, x)$ of target and projectile wells (upper and lower panels, respectively). The coupled-channels calculation does not reproduce properly the exact result: only their magnitudes are in agreement and, in the case of the target continuum, the main structure is reproduced with the maximum and minimum values placed at similar momenta. Anyway, the similar order of magnitude of the target and projectile distributions suggests the importance of including the continuum of all the possible subsystems of the outgoing particles to properly describe all the reaction channels. Moreover they emphasize the fact that, when both the projectile and target systems are weakly bound, the choice for the continuum representation is not obvious and might strongly affect the final result. We notice also that, since both bases are not mutually orthogonal, including both representations leads to overcompleteness, similar to what is done in the Faddeev formalism [31].

To further improve the coupled-channels solution we should add more continuum pseudostates and enlarge the range over which they are defined. Doing so, the two bases would overlap and we would obtain a nonunitarity solution (as we detailed in the previous section). To avoid that, we should let the projectile evolve further, but in this way the continuum component of the system wave function would get away from the two wells and none of the two continuum representations would describe properly the process. As one can notice, already in the $\mathrm{CC}^{(3)}$ calculation a small component of the system wave function has not been included in the range covered by the two bases [around $-30 \mathrm{fm}$ in Fig. 6(d)]. Anyway, even if the exact result is not fully reproduced, the 


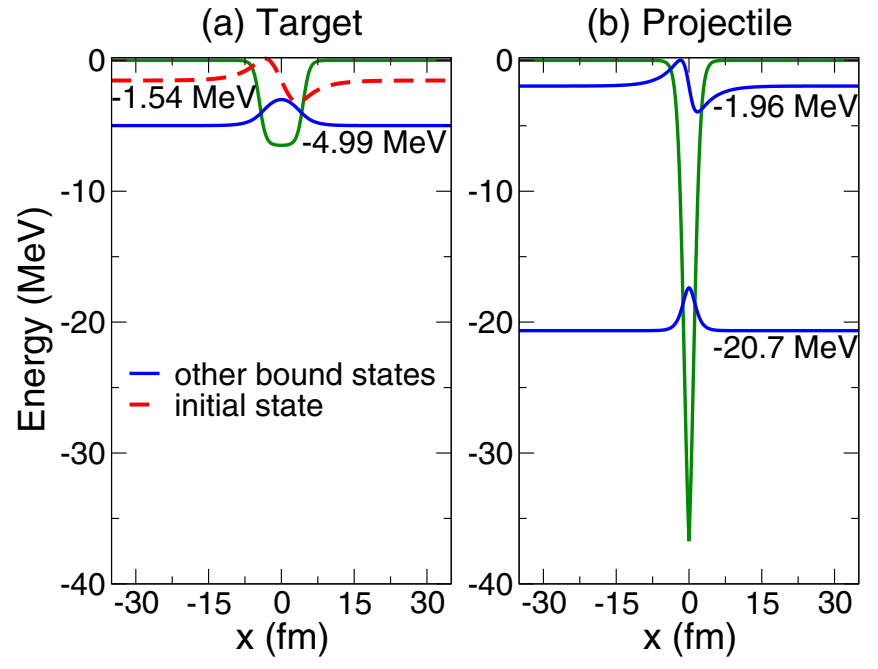

FIG. 9. Target (a) and projectile (b) Woods-Saxon potentials for the case $\mathrm{C}$. Their bound states are schematically depicted in correspondence with their binding energy. The initial state, in this case the target excited state, is the dashed red curve.

coupled-channels calculation allows us to infer important information about the reaction mechanism for this case, and in particular about the transfer process. This resembles the case involving weakly bound systems, in which breakup becomes an important reaction channel and the CDCC description becomes challenging. Also in the three-dimensional case, the discrete basis representing the projectile and/or target continuum is spatially constrained by the extension of the basis, and hence the calculated breakup observables are expected to be reliable only in those kinematical situations which are sensitive to that region [45].

\section{Case C}

We treat now a case in which all the possible channels are relevant: elastic, inelastic, transfer, and breakup. The target and projectile potentials are depicted in Fig. 9 with their respective bound-state wave functions. The initial state is represented by the red dashed curve corresponding to the first excited state of the target. We assume that the target ground state is Pauli forbidden and the corresponding channel should not be included in the calculation. However, in this case we take into account this channel, calling it "decay to g.s." The projectile follows the trajectory (2) with an asymptotic velocity of $0.05 \times c$ corresponding to an incident energy of 1.0 MeV, and with a reduced mass of $0.975 \mathrm{amu}$; the time evolution is divided in $\tau=1450$ steps.

The evolution of the exact wave function during the collision is presented in Fig. 10. The initial wave function exhibits a node, because the initial state corresponds to the first excited state of the well. At the end of the time evolution [Fig. 10(d)] the component related to the target is asymmetric, and so we expect a probability to deexcite the target to its ground state. One can also clearly identify a continuum component [in the region around $-50 \mathrm{fm}$ in Fig. 10(d)], and the presence of transfer to the projectile excited state because the

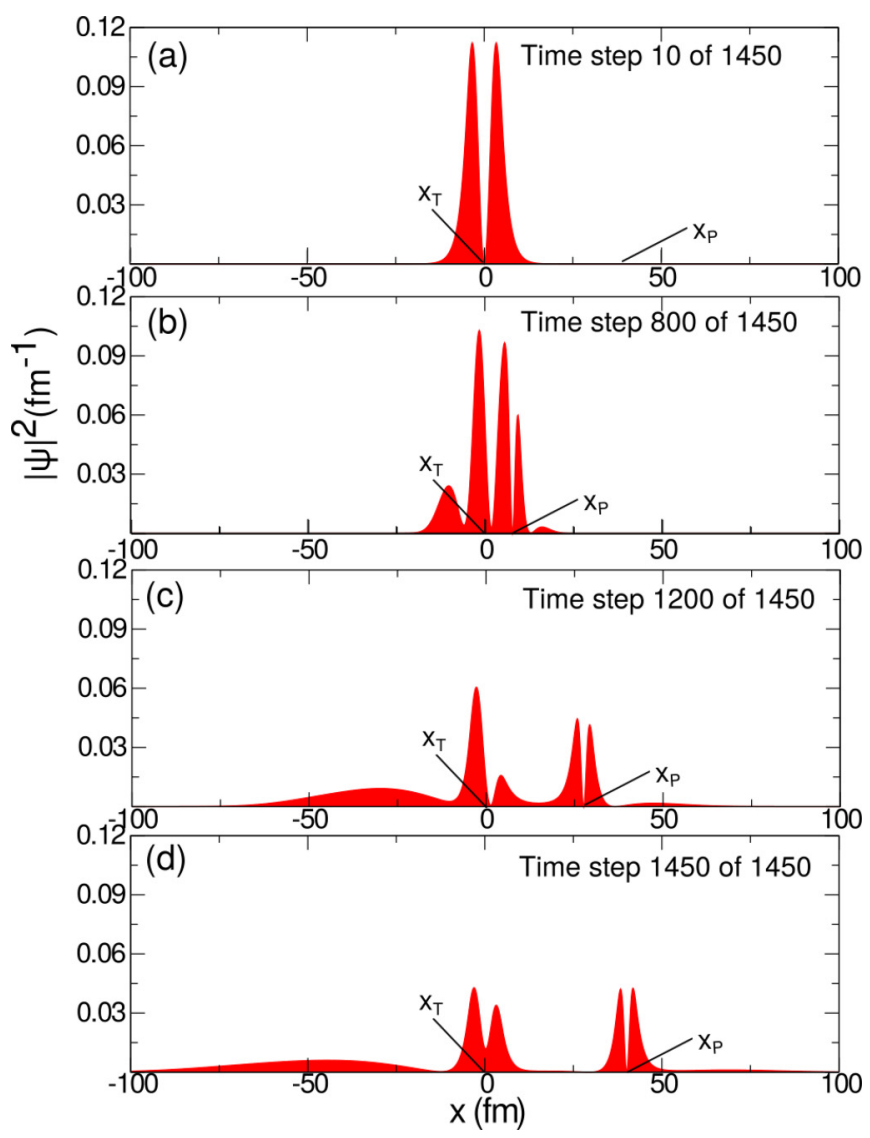

FIG. 10. Model case C, with the exact squared total wave function at different times. Target and projectile potential wells position is indicated by the labels $x_{T}$ and $x_{P}$, respectively. The elapsed time for each frame is also indicated: this process is composed by 1450 time steps; the turning point $t=0$ ps occurs at step 725 .

wave-function component which remains bound to that well presents a node.

The results for this case are reported in Table III, and correspond to the following calculations.

(1) $\mathrm{CC}^{(1)}$ : Coupled-channels calculation in which the target and projectile bound states are included.

(2) $\mathrm{CC}^{(2)}$ : Coupled-channels calculation in which the target bound states and ten target continuum states within the $[-100 ; 100]-f m$ range are included.

(3) $\mathrm{CC}^{(3)}$ : Coupled-channels calculation using the target and projectile bound levels plus the first ten continuum pseu-

TABLE III. Final probabilities for the model case $\mathrm{C}$ obtained with the exact and the coupled-channels methods. Note that $\mathrm{CC}^{(3)}$ and $\mathrm{CC}^{(4)}$ total probability exceeds $100 \%$, as expected.

\begin{tabular}{lccccc}
\hline \hline & Exact & $\mathrm{CC}^{(1)}$ & $\mathrm{CC}^{(2)}$ & $\mathrm{CC}^{(3)}$ & $\mathrm{CC}^{(4)}$ \\
\hline Decay to g.s. & $6.7 \%$ & $0.85 \%$ & $23.5 \%$ & $16 \%$ & $3 \%$ \\
Elastic & $29 \%$ & $99.06 \%$ & $44.8 \%$ & $10 \%$ & $84 \%$ \\
Transfer g.s. & $1.2 \%$ & $0.03 \%$ & & $0.6 \%$ & $0.04 \%$ \\
Transfer 1 & $15.6 \%$ & $0.06 \%$ & & $36 \%$ & $0.2 \%$ \\
Breakup & $47.5 \%$ & & $31.7 \%$ & $44 \%$ & $20 \%$ \\
\hline \hline
\end{tabular}


dostates of the target potential, obtained within a radius equal to the maximum radius of the grid. It corresponds to an energy cutoff in the continuum of $0.3 \mathrm{MeV}$.

(4) $\mathrm{CC}^{(4)}$ : Coupled-channels calculation using the target and projectile bound levels plus the first ten continuum pseudostates of the projectile potential, obtained in a range equal to the maximum radius of the grid. It corresponds to an energy cutoff in the continuum of $0.3 \mathrm{MeV}$.

As evident from $\mathrm{CC}^{(1)}$, the inclusion of the transfer channel bound states is not enough and one needs the inclusion of the continuum, thus indicating that this is not a one-step process in this case. From the $\mathrm{CC}^{(2)}$ calculation we understand that the inclusion of the target continuum allows us to reproduce reasonably well the elastic and breakup channels, as well as the decay to the target ground state, but of course the transfer description is missing because the projectile basis is not included. In this case in which all the reaction channels are relevant, both target and projectile bases including continuum states should be considered in our approximate calculation. However, in this case we have difficulties to apply the same method for the inclusion of the continuum in coupled-channels calculation, as was done in the previous cases. In case A the initial parameters were set in order to give a very small transfer probability to the projectile states, thus the inclusion of target states was enough to reproduce the exact results. In case B the breakup component did not escape quickly from the collision area, so at the end of the time evolution it was localized around the target in an interval that did not overlap with the projectile potential. This fact allowed us to construct two bases, for target and projectile, respectively, which did not overlap at the end of the calculation, thus restoring the probability unitarity. In the case under study, the breakup channel has a strong influence because it is traveling faster than the projectile, and its component is not easily localizable within the spatial grid. What we propose here is to use continuum states which are defined over all the spatial range. This is certainly closer to what the real continuum is (even neglecting the phase shifts due to one of the wells), but surely will not lead to a unitary solution, because of overlap between bound states of a potential well and continuum pseudostates of the other. By including a few target continuum states $\left(\mathrm{CC}^{(3)}\right)$, e.g., ten pseudostates, the deviation from unitarity is not so large, and we are still able to reproduce all the reaction channels. The fact that the inclusion of projectile pseudostates $\left(\mathrm{CC}^{(4)}\right)$ does not reproduce the exact results tells us that the breakup component is mostly influenced by the target potential. Since the projectile well is moving, the overlap between pseudostates and bound states is changing, so we do not expect the coefficients associated to continuum pseudostates to converge to a fixed value without oscillations. In Fig. 11 we show the time evolution of the probabilities computed with the $\mathrm{CC}^{(3)}$ calculation (dotted lines). Each panel corresponds to a given reaction channel; from the lowest, decay to target ground state (green), elastic scattering (blue), transfer to projectile ground state (red), transfer to projectile first excited state (orange), and breakup (black). The comparison with the exact result (solid lines) is presented. Note how the coupled-channels calculation clearly loses unitarity close to the turning point at $t=0 \mathrm{ps}$. By applying this

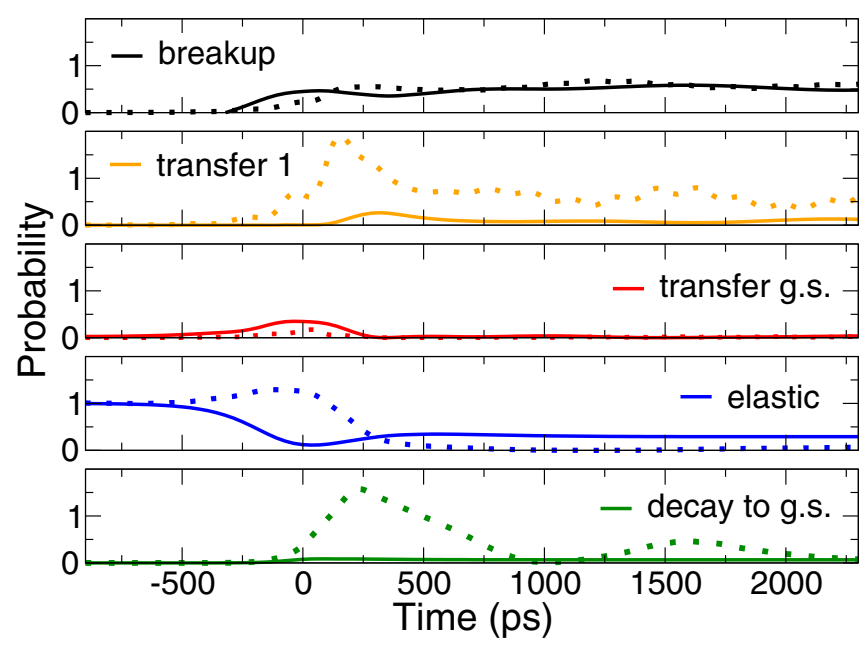

FIG. 11. Evolution of probability as a function of time for the model case $\mathrm{C}$. Each panel corresponds to a reaction channel indicated in the corresponding legend. Solid lines represent the exact results; dotted lines correspond to the results obtained with coupled-channels calculation $\mathrm{CC}^{(3)}$ which are reported in Table III.

method we observe an acceptable description of the breakup channel probability by the coupled-channels calculation with continuum pseudostates, that oscillates around the expected exact value. This is also confirmed by Fig. 12, where we show how the final wave function for the $\mathrm{CC}^{(3)}$ (dashed blue) calculation reproduces reasonably well the breakup component of the exact one (solid red), while the $\mathrm{CC}^{(2)}$ (dot-dashed magenta) fails. However, the effect of the inclusion of two nonorthogonal bases in the $\mathrm{CC}$ description of case $\mathrm{C}$ is evident in Fig. 13, where the projections of the exact (solid red) and $\mathrm{CC}^{(3)}$ (dashed blue) wave functions onto the exact continuum $\varphi(k, x)$ of target and projectile wells (upper and lower panels, respectively) are not in agreement.

The situation presented in this case in which all the reaction channels are competing goes beyond the expected scope of ap-

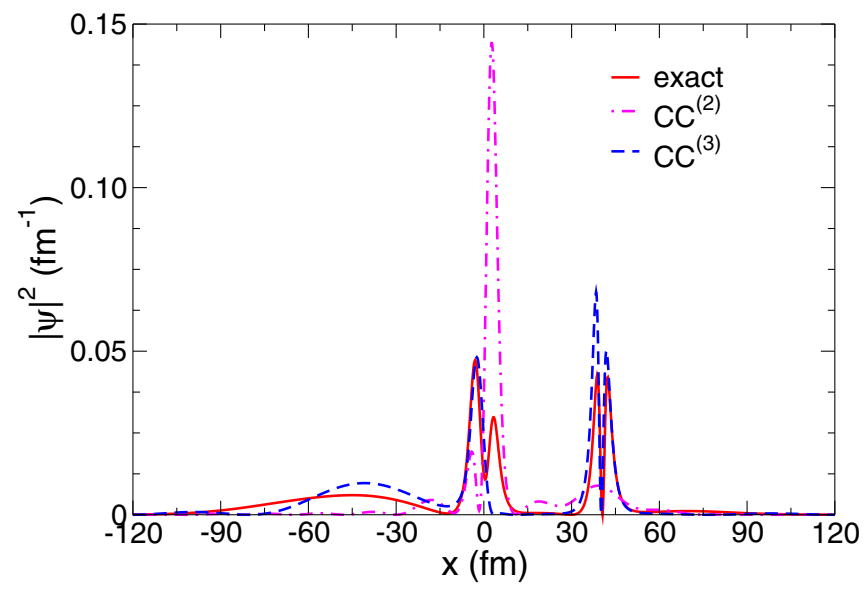

FIG. 12. Final exact wave function (solid red line), final $\mathrm{CC}^{(2)}$ wave function (dot-dashed magenta line), and final $\mathrm{CC}^{(3)}$ wave function (dashed blue line). The projectile potential is placed at $x_{P}\left(t_{f}\right)=$ $40.5 \mathrm{fm}$. 

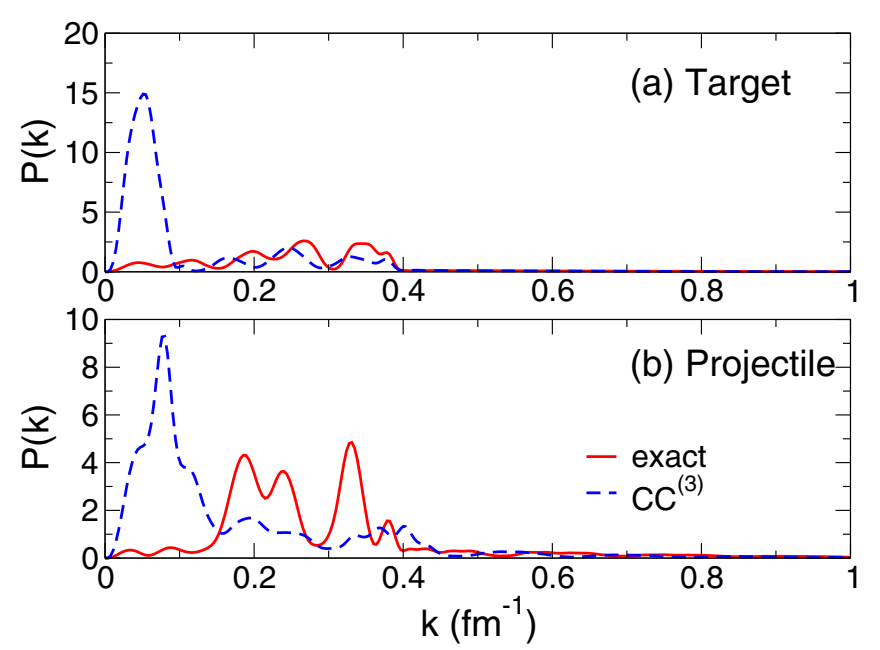

FIG. 13. Breakup probabilities as a function of the momentum in the continuum. The upper and lower panels correspond to the projection on target and projectile states, respectively. Solid red lines indicate the exact final wave function, while dashed blue lines indicate the $\mathrm{CC}^{(3)}$ final one.

plicability of standard coupled-channels methods. In fact, this situation resembles the case in which the distance between the fragments following the projectile breakup $(|\mathbf{r}|)$ is comparable to the target-projectile distance $(|\mathbf{R}|)$. The Faddeev formalism should be better applied [31].

To obtain a proper description of the breakup we should use a set of continuum states which takes into account the effect of the two potential wells together. We have constructed a set of pseudostates diagonalizing in the BOX basis the system Hamiltonian from Eq. (1), including both potentials at time $t=t_{f}$ and using the whole spatial grid. We have then projected the final exact wave function onto this set of continuum states to evaluate how they describe the breakup channel probability. In Table IV we show this probability varying the number of pseudostates included in the basis. Increasing $N$, the total probability tends to the exact result. We need the contribution of a large number of states to get the proper result.

In a time-dependent model, to include the continuum of all the constituents at the same time one should compute a complete basis (including bound and continuum pseu-

TABLE IV. Breakup probabilities for the model case $\mathrm{C}$ obtained with the projection of the final exact wave function onto $N$ continuum pseudostates calculated taking into account both potential wells in the final configuration.

\begin{tabular}{cc}
\hline \hline$N$ & Breakup probability \\
\hline 30 & $17.8 \%$ \\
50 & $41.6 \%$ \\
55 & $43.5 \%$ \\
60 & $46.7 \%$ \\
62 & $47.3 \%$ \\
63 & $47.5 \%$ \\
Exact result & $47.5 \%$ \\
\hline \hline
\end{tabular}

dostates) for the total system at each time step. This follows the so-called two-center method, widely used in atomic physics and recently applied for nuclear physics calculations [46-48].

It is worth mentioning here that a difference with respect to the usual calculation is that in our framework we only included real potentials: the use of an imaginary component can account for the fusion and other excluded channels and could solve the nonunitary issue of the present formalism. This solution could also account for the neglected target recoil in our model. In our model the trajectory is fixed and the target recoil is neglected, so the energy is not conserved in these processes. However, this is not expected to affect the result, when the asymptotic energy is much higher than the excitation one. In the situation shown in the last case (Sec. III C), the excitation energy might be a significant fraction of the available kinetic energy or even be of the same order. This resembles the limitations encountered in the eikonal model [49] (which is based on the assumption of straight projectile trajectories) when it is applied to study reactions at low beam energy [50-52]. Even if we used real potentials, the main conclusions on the role of the continuum in the reaction mechanism would remain unchanged. The description of a continuum using a basis which takes into account the phase shift induced by only one potential well and, more in general, the choice of the continuum which describes only a subsystem of the reaction constituents are not good approximations in the case of a dominant breakup channel.

\section{CONCLUSIONS}

We studied processes involving both bound and weakly bound systems, and all the possible channels of a direct reaction within a simple one-dimensional problem consisting in the scattering of a particle, initially bound in a potential well (the target), by another moving well (the projectile). An appealing feature of this problem is that it can be solved "exactly" using the time-dependent Schrödinger equation, thus providing a robust benchmark for approximate methods, such as the popular coupled-channels formalism employed in atomic, molecular, and nuclear physics. In this framework, we can investigate the role of the continuum, which is found to be relevant even for situations in which the considered particle is initially in a well-bound state. In particular, we address the issue of the inclusion of a discretized continuum in a coupled-channels time-dependent calculation. Continuum waves are treated in our computational model in "mathematical" representations of nonorthogonal sets of normalizable wave functions that reflect the phase shifts induced by each potential well. We observe that when the breakup channel is almost negligible with respect to the other direct reaction channels, like our case A, this representation of the continuum works well. When the breakup clearly dominates or is competing with the other channels, like in cases $\mathrm{B}$ and $\mathrm{C}$, the inclusion of a set of continuum pseudostates associated to only one of the potential wells is not enough to properly describe the reaction. However, the spatial limitations to the continuum might give only a partial description of the process. So, the inclusion of the continuum in computational 
models is always an artifact, and in many cases the results depend on how the continuum is constructed, as clearly shown in one dimension. Therefore, the discretized continuum defined taking into account both potential wells on the whole range should be the best approximation. To take into account this configuration in a time-dependent picture in the present formalism is, however, not possible, so we suggest the solution of the "two-center" method [46-48]. The natural extension of this paper would be then the development of a continuum-discretized coupled-channels calculation within the two-center formalism, to be compared to the exact solution of the time-dependent Schrödinger equation.

The present framework could also be applied to the description of direct reactions involving two-neutron halo nuclei, e.g., to study the role of pairing between the two valence particles. First results for the exact solution of this process can be found in Refs. [27,53,54].

\section{ACKNOWLEDGMENTS}

We thank Prof. Alexis Diaz-Torres for a careful reading of the paper, helpful discussions, and comments. L.M. is grateful to Università degli Studi di Padova and Universidad de Sevilla, where the majority of this project has been developed under Istituto Nazionale di Fisica Nucleare (Padova) financial support. L.M. is supported by United Kingdom Science and Technology Facilities Council (STFC) under Grant No. ST/L005743/1.
[1] I. Tanihata, H. Hamagaki, O. Hashimoto, Y. Shida, N. Yoshikawa, K. Sugimoto, O. Yamakawa, T. Kobayashi, and N. Takahashi, Phys. Rev. Lett. 55, 2676 (1985).

[2] I. Tanihata, T. Kobayashi, O. Yamakawa, S. Shimoura, K. Ekuni, K. Sugimoto, N. Takahashi, T. Shimoda, and H. Sato, Phys. Lett. B 206, 592 (1988).

[3] S. G. Nilsson, Mat. Fys. Medd. Dan. Vid. Selsk. 29, 16 (1955).

[4] B. R. Mottelson and S. G. Nilsson, Mat. Fys. Skr. Dan. Vid. Selsk. 1, 8 (1959).

[5] K. Heyde, Basic Ideas and Concepts in Nuclear Physics: An Introductory Approach (IOP, Bristol, UK, 1999).

[6] I. Tanihata, J. Phys. G: Nucl. Part. Phys. 22, 157 (1996).

[7] I. Tanihata, H. Savajols, and R. Kanungo, Prog. Part. Nucl. Phys. 68, 215 (2013).

[8] I. Tanihata, Progr. Part. Nucl. Phys. 35, 505 (1995).

[9] G. R. Satchler, Introduction to Nuclear Reactions (Macmillan, New York, 1980).

[10] G. Rawitscher, Phys. Rev. C 9, 2210 (1974).

[11] N. Austern, Y. Iseri, M. Kamimura, M. Kawai, G. Rawitscher, and M. Yahiro, Phys. Rep. 154, 125 (1987).

[12] I. J. Thompson and F. M. Nunes, Nuclear Reactions for Astrophysics: Principles, Calculation and Applications of LowEnergy Reactions (Cambridge University, Cambridge, England, 2009).

[13] M. Yahiro, K. Ogata, T. Matsumoto, and K. Minomo, Progr. Theor. Exp. Phys. 2012, 01A206 (2012).

[14] T. Sawada and K. Thushima, Progr. Theor. Phys. 76, 440 (1986).

[15] Z. C. Kuruoğlu, Phys. Rev. C 43, 1061 (1991).

[16] N. J. Upadhyay, A. Deltuva, and F. M. Nunes, Phys. Rev. C 85 , 054621 (2012).

[17] W. Du, P. Yin, Y. Li, G. Chen, W. Zuo, X. Zhao, and J. P. Vary, Phys. Rev. C 97, 064620 (2018).

[18] M. Boselli and A. Diaz-Torres, Phys. Rev. C 92, 044610 (2015).

[19] J. Révai, Nucl. Phys. A 438, 512 (1985).

[20] B. Milek, R. Reif, and J. Révai, Phys. Lett. B 150, 65 (1985).

[21] Note that, since we do not simulate Coulomb barriers, we assume the valence particle to be a neutron.

[22] H. Esbensen, R. A. Broglia, and A. Winther, Ann. Phys. (NY) 146, 149 (1983).

[23] Bound states are obtained by solving the finite-difference method on the whole grid [55].
[24] L. Moschini, F. Pérez-Bernal, and A. Vitturi, J. Phys. G: Nucl. Part. Phys. 43, 045112 (2016).

[25] P. Bonche, S. Koonin, and J. W. Negele, Phys. Rev. C 13, 1226 (1976).

[26] We are also imposing vanishing boundary conditions.

[27] L. Moschini, Ph.D. thesis, Università degli Studi di Padova and Universidad de Sevilla, 2017.

[28] R. A. Broglia and A. Winther, Nucl. Phys. A 182, 112 (1972).

[29] R. A. Broglia and A. Winther, Phys. Rep. C 4, 154 (1972).

[30] K. Dietrich and K. Hara, Nucl. Phys. A 211, 349 (1973).

[31] L. D. Faddeev, Zh. Eksp. Teor. Fiz. 39, 1459 (1960).

[32] L. Moschini, J. Phys.: Conf. Ser. 566, 012027 (2014).

[33] A. Vitturi and L. Moschini, J. Phys.: Conf. Ser. 590, 012007 (2015).

[34] L. Moschini, A. Vitturi, and A. Moro, in Basic Concepts in Nuclear Physics: Theory, Experiments and Applications, edited by J.-E. García-Ramos, C. E. Alonso, M. V. Andrés, and F. Pérez-Bernal (Springer, New York, 2016), pp. 181-183.

[35] L. Moschini, A. Vitturi, and A. Moro, J. Phys.: Conf. Ser. 981, 012004 (2018).

[36] F. Flavigny, A. Obertelli, A. Bonaccorso, G. F. Grinyer, C. Louchart, L. Nalpas, and A. Signoracci, Phys. Rev. Lett. 108, 252501 (2012).

[37] A. M. Moro, R. Crespo, F. Nunes, and I. J. Thompson, Phys. Rev. C 66, 024612 (2002).

[38] J. Lubian, T. Correa, E. F. Aguilera, L. F. Canto, A. GomezCamacho, E. M. Quiroz, and P. R. S. Gomes, Phys. Rev. C 79, 064605 (2009).

[39] N. Keeley, N. Alamanos, K. Kemper, and K. Rusek, Progr. Part. Nucl. Phys. 63, 396 (2009).

[40] K. Hagino, A. Vitturi, C. H. Dasso, and S. M. Lenzi, Phys. Rev. C 61, 037602 (2000).

[41] A. Diaz-Torres and I. J. Thompson, Phys. Rev. C 65, 024606 (2002).

[42] L. F. Canto, J. Lubian, P. R. S. Gomes, and M. S. Hussein, Phys. Rev. C 80, 047601 (2009).

[43] F. M. Nunes and I. J. Thompson, Phys. Rev. C 59, 2652 (1999).

[44] J. Lei and A. M. Moro, Phys. Rev. Lett. 123, 232501 (2019).

[45] A. Deltuva, A. M. Moro, E. Cravo, F. M. Nunes, and A. C. Fonseca, Phys. Rev. C 76, 064602 (2007).

[46] A. Diaz-Torres and W. Scheid, Nucl. Phys. A 757, 373 (2005). 
[47] A. Diaz-Torres, Phys. Rev. Lett. 101, 122501 (2008).

[48] A. Diaz-Torres, Comp. Phys. Comm. 224, 381 (2018).

[49] R. Glauber, in Lectures in Theoretical Physics, edited by W. Brittin and L. Dunham (Interscience, New York, 1959), Vol. 1, p. 315 .

[50] C. E. Aguiar, F. Zardi, and A. Vitturi, Phys. Rev. C 56, 1511 (1997).
[51] C. Hebborn and P. Capel, Phys. Rev. C 96, 054607 (2017).

[52] C. Hebborn and P. Capel, Phys. Rev. C 98, 044610 (2018).

[53] A. Vitturi, L. Moschini, K. Hagino, and A. M. Moro, AIP Conf. Proc. 1681, 060001 (2015).

[54] A. Vitturi and L. Moschini, J. Phys.: Conf. Ser. 966, 012045 (2018).

[55] C. A. Moyer, Comp. Sci. Eng. 8, 32 (2006). 